\title{
A Network Aggregation Tool for the Energy System Modelling Framework Spine
}

\author{
Iasonas Kouveliotis-Lysikatos, Manuel Marin \\ Electrical Engineering and Computer Science \\ KTH - Royal Institute of Technology \\ Stockholm, Sweden \\ \{iasonas, manuelma\}@kth.se
}

\author{
Jon Olason \\ Digital tools Dept. \\ Svenska Kraftnät \\ Stockholm, Sweden \\ jon.olason@svk.se
}

\author{
Mikael Amelin, Lennart Söder \\ Electrical Engineering and Computer Science \\ KTH - Royal Institute of Technology \\ Stockholm, Sweden \\ \{amelin, lsod $\} @$ kth.se
}

\begin{abstract}
Network reduction techniques are very useful for taming the complexity of simulating large interconnected power systems. In this paper, we present the implementation of a tool that performs a multi-area spatial network aggregation based on the generalized Radial Equivalent Independent (REI) methodology. The tool is integrated in Spine, an open source energy modelling framework currently under development. The main objective of Spine is to enable open, practical, flexible and realistic planning of future European energy grids. The validity and the efficacy of the reduction tool is investigated through a series of power flow scenarios for various test networks.

Index Terms-network reduction, energy system modelling, open source software, Spine.
\end{abstract}

\section{NOMENCLATURE}

$G_{a} \quad$ Set of the aggregated generation buses of area $a$.

$N \quad$ The number of nodes of the original network.

$N_{a} \quad$ Set of the aggregated load buses of area $a$.

$S_{i} \quad$ Complex power injection of node $i$.

$V_{i} \quad$ Complex voltage of node $i$.

$Y_{i, j} \quad$ Admittance of line connecting nodes $i$ and $j$.

$Y_{\text {New }}$ Admittance matrix of expanded network area.

$Y_{\text {Red }} \quad$ Reduced admittance matrix of network area.

\section{INTRODUCTION}

\section{A. Motivation and Background}

The ever-increasing complexity of modern power systems calls for novel methodologies to provide efficient yet accurate estimates of the grids' operational aspects, without requiring the full analytical modelling of their infrastructure. Aggregated models can be used for efficiently producing such estimates, to be exploited next within a wide variety of operational and planning power system studies [1]. Recently, open source software for energy modelling studies is increasingly adopted both from the academia and the industry. Their community-driven development, and their capability to perform and transparently reproduce energy models and case studies are some of their valuable qualities, especially when it comes to proposing and arguing about new energy policies.

This project has received funding from the European Union's Horizon 2020 research and innovation programme under grant agreement N. 774629.

\section{B. Relevant Literature}

In terms of spatial aggregation, the REI methodology constitutes a well-studied practice for producing a reduced equivalent of a power flow model [2]. The multi-area REI equivalent aims at efficiently calculating estimates of the interchanges between different grid areas, separated due to company boundaries or by geographical regions. In later works, the method has been enhanced and applied in reducing the size of the original power flow model of a power system, in order for it to become more tractable for the calculation of the spinning reserve [3] under a Security Constraint Unit Commitment (SCUC) setup, or for planning studies which require the assessment of large number of scenarios due to the stochastic nature of renewable generation units [4].

Amongst the different variations of the REI methodology, a common step that is performed is the partitioning of the system nodes into a number of areas. In order to better tune the reduced model, the partitioning method should be chosen according to the specific requirements of the case study [5]. A basis for power network partitioning is using the admittance matrix or variations of it as a distance metric (i.e. the electrical distance), but other objectives might be set such as the minimization of active and reactive power imbalances between generation and load within the defined clusters [6]. After the partitioning has been performed, the system areas are modelled by REI circuits, whose appropriate combination leads to an aggregated model of the initial system. Different reduction approaches comprise the fitting of a Power Transfer Distribution Matrix (PTDF) of reduced dimension using nonlinear optimization techniques [7], or the estimation of the electrical parameters of aggregated nodes and their inter-area interconnections [8].

Various open source energy modelling software exist that enable the development and assessment of energy modelling studies, such as OSeMOSYS [9], TIMES, [10] and the open energy modelling framework, oemof [11].

\section{Contributions and Organization}

However, for effectively conducting multi area power system studies, there is a value in using open-source software and tools able to reduce their computational complexity. In this paper, we present the design and implementation of a 
set of components extending the Spine framework that create and visualize the reduced power flow model of a power system. Moreover, we enhance the multi-area REI methodology combining it with a fast electrical distance calculation method for the network clustering, something which has not been proposed before. Also, we introduce a novel mapping of abstract power system components to the modelling conventions of Spine in order to accommodate AC/DC power flow models and lastly, we evaluate the enhanced multi-area REI method by investigating the method in terms of accuracy and performance.

\section{SPINE FRAMEWORK}

\section{A. Spine Model, Toolbox and Data Structure}

The Spine framework ${ }^{1}$ is a high-level software application that enables and facilitates the modelling and execution of multi-energy case studies [12]. The framework consists of two main components, the Spine Toolbox and the Spine Model. The Spine Toolbox is a sort of shell that enables the definition and management of various energy systems models, including Spine Model itself, which is a dedicated tool for multivector energy system modelling. In this way, Spine Toolbox encompasses Spine Model while enabling a set of ancillary tools for data gathering, representation, persistent storage and visualization as well as the orchestration of the execution and validation of complex scenarios. Spine Toolbox provides the ontology for developing new applications and linking them with Spine Model.

In order to realize all the previous, the Spine data structure provides the common vocabulary. Available importers and exporters can be used for converting between the model representation of various popular optimization software, such as GAMS. Software and user interfaces enable the integration of the various components and facilitate the model construction and validation by the end-user. Spine is developed in its entirety using open source software and libraries. Specifically, Spine toolbox is implemented in the Python programming language, while Spine Model is implemented in Julia. A highlevel diagram of Spine is provided in Fig. 1, depicting the components described next.

The Spine software application spreads over two execution environments, appropriately interlinking and orchestrating them, and providing the necessary software interfaces for accommodating custom (or even proprietary) software modules. The Spine model (Fig. 1 left), is executed in a Julia environment, while the other core Spine functionalities and services such as the Spine data store, the Spine Database API, and the data visualization and clustering components live in a Python execution environment (Fig. 1 right). Spine is bundled with a friendly user interface developed in the cross-platform graphics library Qt and accessible in Python via the PySide2 module.

\footnotetext{
${ }^{1}$ https://github.com/Spine-project
}

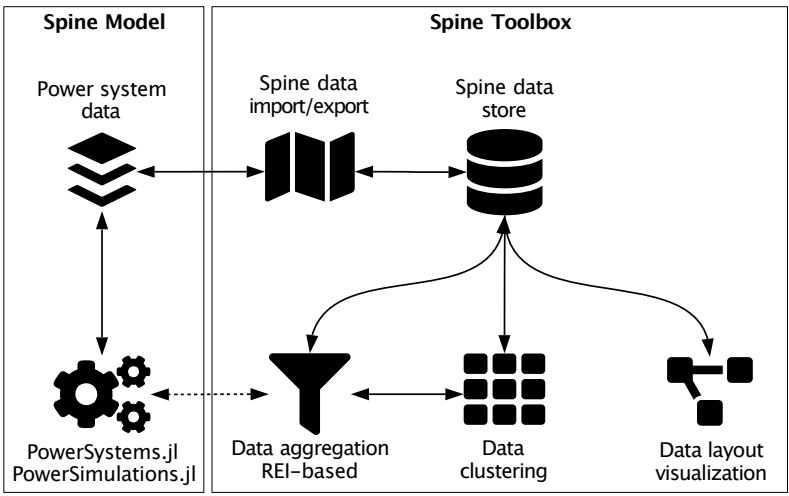

Fig. 1. Spine conceptual diagram and new components.

\section{B. The Spine Model Building Blocks}

The Spine data structure is designed using a generic Entity - Attribute - Value approach with Classes and Relationships (in short EAV/CR), tailored to represent multi-vector energy systems. The abstract classes and the relationships between them enable the formulation of a multi-commodity, flowbased model of a generic energy system, in an object-oriented manner. The main abstract classes are described below [13]:

- Node: a point in space where energy from a certain vector gets balanced.

- Unit: a device, installed between two nodes, capable of converting energy from one vector to another.

- Connection: a device, also installed between two nodes, capable of transferring energy within the same vector.

- Commodity: a type of energy flow, uniquely associated with a vector.

- Temporal block: a period of time with a specific resolution.

\section{Power System Models IN SPINE}

\section{A. Integration of Power Flow Models}

The flow-based representation of the energy vectors might be adequate for simulating hydro-power or district heating studies, but could be deemed simplistic for Power System analysis studies. For this reason, better approximations are being explored and developed as a way to integrate the electricity flows in Spine model. Three alternatives that a modeller can follow to simulate electricity flows at this point of Spine development are listed below:

- By using PowerModels.jl Julia package [14], directly in SpineModel.jl,

- Alternatively, by using PowerSystems.j1 and Powersimulations.jl Julia packages [15] in Spinemodel.jl (that also depend on PowerModels.jl),

- By using external (custom) Python scripts/modules which perform power flow studies (for specific applications) and uses in turn Python-based power system modelling libraries such as the PyPower, or PandaPower modules [16]. 


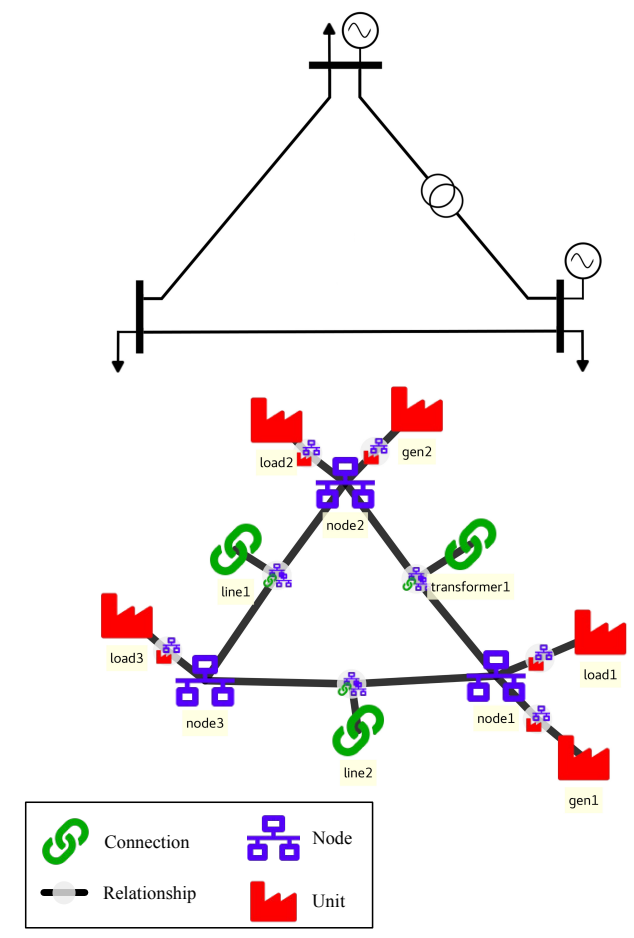

Fig. 2. A mapping of power system components to the Spine data structure.

To enable the representation of power grids within the Spine data store in any of the above options, the various power system components (transmission/distribution lines, transformers, buses, etc.) need to be mapped into the Spine abstract classes (node, unit, connection, etc.). This mapping procedure can be easily handled, parameterized, and replicated by the Spine importing/exporting tools. Such a mapping is shown in Fig. 2, associating the fundamental power system components for building a single-phase power-flow model (top), to the building blocks of Spine data structure (bottom). Different mapping approaches can be adopted by the user however, according to the specific nature of each case study. Having acquired a complete and concise data model in the specific Spine data structure, the user has two alternatives for compiling power flow related models, depending on the requirements of the system that is modeled, as described below.

a) Representing Power Flow Equations Internally in Spine Model: In the case that a co-simulation of the electricity with other energy carriers is required, the user only needs to define certain parameters of the Spine objects and relationships (the ones corresponding to power systems components). Then, at Spine Model building time, the relevant parameters will trigger the insertion of additional variables and constraints (adding also supplementary terms for the objective function - if it is required). Since both Spine model and PowerModels.jl depend on the JuMP library for formulating the optimization model, the SpineModel.jl can easily be extended by adding the required power flow equations, in accordance with the defined mapping of the power system components to the Spine data structure. Clearly, the multitude of different power flow formulations (and relaxations) of PowerModels.jl and PowerSystems.jl packages - each one suitable for different categories of power system analysis studies, are available for the user to choose from, showcasing the versatility, modularity and extensibility of Spine.

b) Using External Power System Analysis Tools: For simpler (or more specialized) modeling cases, the user can utilize the Spine data structure, workflow and visualization components, by introducing custom Python (or Julia) modules. In that way, the user modules can access and process the Spine data, redirect it to other components of a Spine workflow, or store it again in a Spine database. Other standardized data formats such as the MATPOWER case format and the PSS/E-PTI, are made available to be represented and processed within Spine software. The important qualities of code reusability and compatibility with various data formats of Spine architecture are hence highlighted. Three custom data processing components originally developed in [2] and [17] are briefly discussed next. The components are developed in Python and use the PyPower module for the power flow calculations. Their integration in Spine toolbox follows the mapping shown in Fig. 2.

\section{SPecialized Components For POWER Systems ANALYSIS AND REDUCTION}

\section{A. The Clustering Technique}

The network partitioning is an important preliminary step before the calculation of the REI model. The implemented component can be customized by the user according to the needs of the specific case study. The basic functionality of the clustering component is based on the definition of a distance metric. A very common distance metric that is used for clustering steady-state power flow models, is the electrical distances metric [5]. The simplest form of an electrical distance matrix is the inverse of admittance matrix $Y_{B U S}$, but certain modifications can lead to better results by taking into account e.g. the current network loading conditions [4]. If the geographical coordinates of the buses are known beforehand, the user can insert them and prompt the clustering function to create the distance metric directly from the topological distances of the buses. This approach, might differ from the electrical distances-based solution, but could be more suitable when the exchanges between regionally-partitioned power systems need to be studied in more detail. If very few or no topological information are available, it is still possible to infer a topology from the admittance matrix of the network. This functionality is provided by the Network Layout Component that is described next.

After the distance metric has been chosen, the nodes are partitioned in different areas, the number of which is empirically set by the user. For the clustering of the buses the kmeans algorithm from the Python package sklearn is employed as a default. The user is able to test different initialization parameters, or algorithmic variations, or even provide a custom module that best fits the needs of the application. The network 
clustering component is embedded in the REI component, but can also be used by other Spine components.

\section{B. The Network Layout Component}

This component generates a topological network layout from the electrical characteristics of the power grid. This functionality is useful for creating two-dimensional spatial visualizations of the network buses and branches. The generated layout might not necessarily catch the original layout of the network, but can quickly provide a draft overview of it, even in the absence of geographical data. If actual coordinates are available (even for a small portion of the buses), the produced layout is able to achieve much better approximates of the original. Another possible usage of this component is to provide the set of coordinates for the buses to be partitioned by the clustering component.

The method computes a distance matrix between all buspairs based on the electrical characteristics of the network and then provides a layout solution [17]. It achieves a parallelization of the computational steps, rendering itself an efficient pre-processing step before the network clustering. The component is developed in Python and exhibits remarkably scalable behavior, being able to cope with very large systems in a few seconds.

\section{The REI Model Calculation Component}

Assuming that the network clustering solution is calculated, the REI methodology dictates substituting each area with an equivalent power flow model. Since the equivalent models can only provide estimates of the full power flow model, a tradeoff is expected between the computational complexity and the accuracy of the reduced model. In general, a larger number of areas should provide better results (naturally leading though to an increased computational complexity) [2]. The generalized procedure for constructing the REI model of a grid's area consists of a number of steps. First, the network buses are separated depending on whether they are connected (essential buses) or not (non-essential by default) with buses from other areas. The non-essential buses' injections are appropriately moved in two newly introduced buses, one aggregating the generators and the other aggregating the loads. The implementation of the specific REI methodology prescribes the way the electrical characteristics of the new buses and lines of the reduced model are calculated. The user is able to adjust certain parameters in order to tune the reduced model to the requirements of their study, or even extend the component. The REI component takes as an input a Spine database of the original system, and a set of parameters, e.g. the network areas (lists containing the buses of each area), etc., and provides as an output a new Spine database of the reduced model. The reduction is performed for each cluster of the initial network based on the REI methodology [18], [2], [3] and is presented briefly next.

For each area a new admittance matrix $Y_{N e w}$ is calculated from the original one with dimensions $(N+2) \times(N+2)$, by introducing two fictitious buses one for aggregating loads (included in set $N_{a}$ ) and one for generation (included in set $G_{a}$ ) - only for the non-essential buses. All generators and loads are transferred to the corresponding newly introduced buses and are connected to the original buses through the respective admittances:

$$
\begin{aligned}
Y_{N+1, j} & =-\frac{S_{j}^{*}}{\left|V_{j}\right|^{2}} \forall j \in G_{a} \\
Y_{N+2, i} & =-\frac{S_{i}^{*}}{\left|V_{i}\right|^{2}} \forall i \in N_{a}
\end{aligned}
$$

$S_{j}^{*}, S_{i}^{*}, V_{j}, V_{i}$ are retrieved from a power flow or an optimal power flow solution of the original network. For the calculation of the newly introduced diagonal elements the following expressions are used:

$$
\begin{aligned}
& Y_{N+1, N+1}=\frac{\sum_{j \in G_{a}} S_{j}^{*}}{\left|V_{N+1}\right|^{2}} \\
& Y_{N+2, N+2}=\frac{\sum_{i \in N_{a}} S_{i}^{*}}{\left|V_{N+2}\right|^{2}}
\end{aligned}
$$

The final step is the calculation of the reduced admittance matrix $Y_{\text {Red }}$ from $Y_{N e w}$ :

$$
\begin{gathered}
Y_{N e w}=\left(\begin{array}{cc}
Y_{E, E} & Y_{E, N} \\
Y_{N, E} & Y_{N, N}
\end{array}\right) \\
Y_{R e d}=\left(Y_{E, E}-Y_{E, N} Y_{N, N}^{-1} Y_{N, E}\right)
\end{gathered}
$$

The matrix partitions $Y_{E, E}$ and $Y_{N, N}$ correspond to the essential buses and non-essential buses respectively and the partitions $Y_{E, N}$ and $Y_{N, E}$ to the connections between nonessential and essential buses.

\section{Results}

In this section, the functionality of each component that was presented previously is explored, employing official IEEE test power networks. Each test network that was used, is available in the MATPOWER and PyPOwer formats and can be easily imported into, and exported from Spine software. In Fig. 3 the IEEE 39-buses transmission system is shown as it was plotted by the network layout component. Specifically, in Fig. 3 (a) both an inferred topology of the network, and the node clustering are presented. The clustering component takes as input the number of areas and provides a clustering solution using as a distance metric the set of coordinates that were calculated for creating the spatial layout. The advantage of this workflow is that the results from the computations performed by the layout component are exploited also by the clustering tool, avoiding the need to calculate an additional electrical distance-based metric.

As regards the REI methodology, the essential buses (border buses in this case) are denoted in all figures with circles with an increased radius compared to the non-essential ones. Different colours are used to indicate the different clusters. In Fig. 3 (b), a layout of the IEEE 39-bus system is presented, as 
it was calculated after the application of the REI methodology. Apart from the essential nodes and the lines between them which remain unaltered, the introduction of the two fictitious nodes for each area can be observed together with the new lines between them and the essential buses. Similar results are provided for the IEEE 118-buses system in Fig. 4 and for the IEEE 300-buses system in Fig. 5, having chosen a number of five and six areas respectively.

The accuracy and the computation time improvement for executing an optimal power flow on the reduced model of the IEEE 118-buses test system is investigated in Table I, by testing different numbers of areas. For evaluating the accuracy of the method, one could calculate the error between all comparable power flow parameters between the initial and the reduced model. In Table I, the total calculated active power generation and the operational cost are compared, while in Table II, the total active power generation error is used as an evaluation metric. What is observed for this implementation of the REI method, is that increasing the number of areas provides more accurate results (for up to a number of areas), but increases in turn the computation time. Specifically, for a number of eight areas the execution of the reduced optimal power flow model is slightly worse than running the full model for the original IEEE 118-buses case. Also, different evaluation metrics might be suitable for tuning the REI method for case studies with different objectives. In Table II, the accuracy and computation time of the reduced power flow models of various test systems is presented, for empirically chosen numbers of areas. It is very interesting to observe that a significant reduction for a much larger network is achieved (the 2000buses test system ACTIVSg2000).

For evaluating the added value of the enhanced REI method, we investigate its performance in terms of accuracy and computational complexity for calculating the reduced power model, by comparing it with a preexisting implementation (i.e. [2]), that performs the network clustering based on the calculation of the electrical distance metric (which requires the inversion of the admittance matrix of the original network). In Fig. 6 (a), the error in the objective value of the optimal power flow solution of the reduced IEEE 118-buses transmission system for both methods is shown (baseline and proposed), for different number of areas, while Fig. 6 (b) presents the comparison in the total required calculation time of the reduced network respectively. In both sub-figures it is observed that the proposed method improves significantly the objective value error, especially for larger number of areas, while requiring a relatively stable time for the calculation of the REI. For a number of two areas the baseline method performs slightly better, but as the number of areas is increased the proposed method significantly outperforms the baseline methodology.

\section{Conclusions}

We have presented and evaluated an enhanced multi area REI-based network reduction tool for the Spine energy modelling framework. We have discussed the different ways to
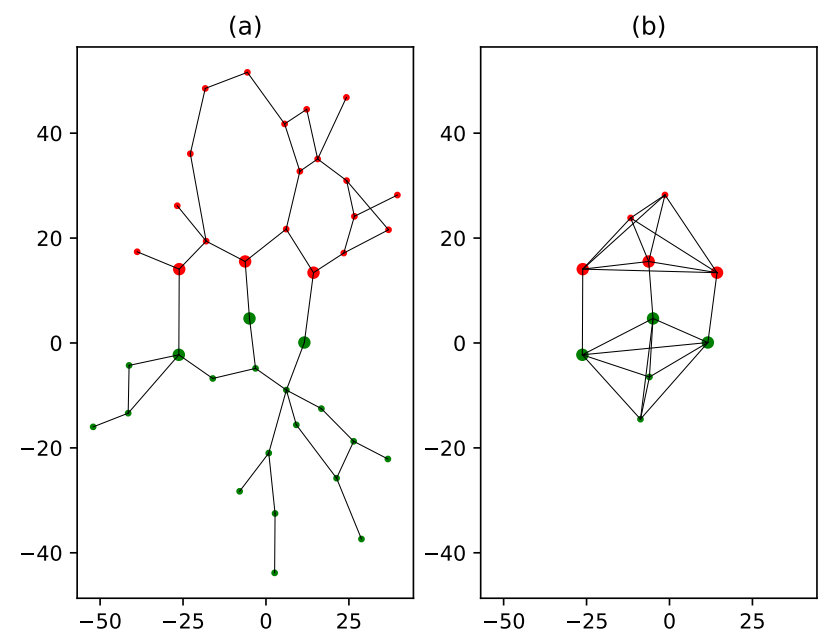

Fig. 3. The IEEE 39-buses transmission system, (a): the original system and the partitioning of the nodes (essential nodes are denoted with circles with increased radius), (b): the reduced system in two areas. (a)

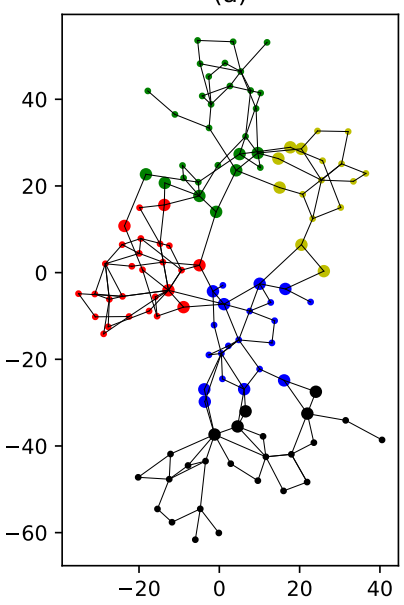

(b)

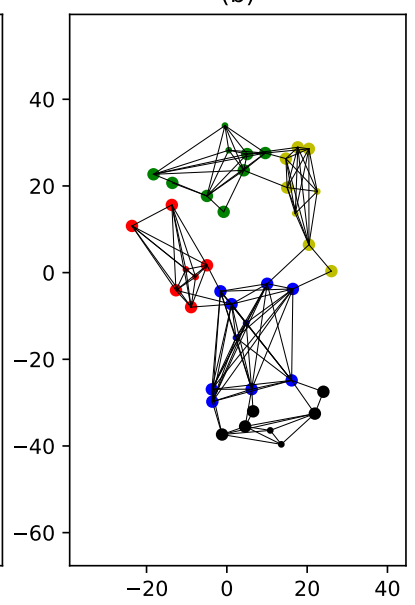

Fig. 4. The IEEE 118-buses transmission system, (a): the original system and the partitioning of the nodes (essential nodes are denoted with circles with increased radius), (b): the reduced system in five areas.

integrate power flow models in Spine energy modelling framework and highlighted its design advantages, e.g. its modularity and extensibility. The efficiency and potential improvement of the reduced power flow models has been assessed for different IEEE test systems, leading to the main result that increasing the number of areas of the reduced power flow model provides a better approximation of the original system (coming with an increased computing time), for up to a certain number of areas where the performance of the reduced models starts to deteriorate. Regarding the evaluation of the proposed reduction technique, it was shown that it improves the error of the reduced model compared to a baseline REI method that uses an electrical distance-based clustering solution, while reducing (in most cases) the calculation time of the reduced power flow model. 
(a)

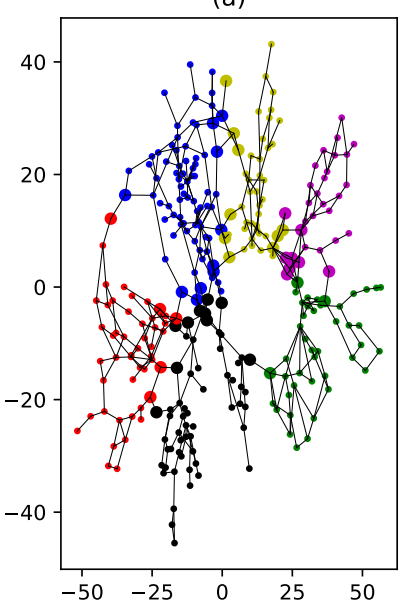

(b)

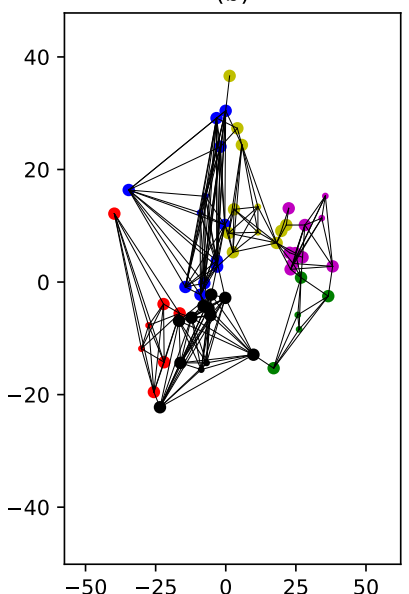

Fig. 5. The IEEE 300-buses transmission system, (a): the original system and the partitioning of the nodes (essential nodes are denoted with circles with increased radius), (b): the reduced system in six areas.

(a)

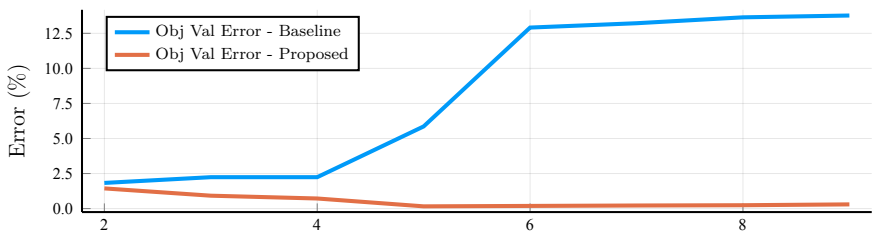

(b)

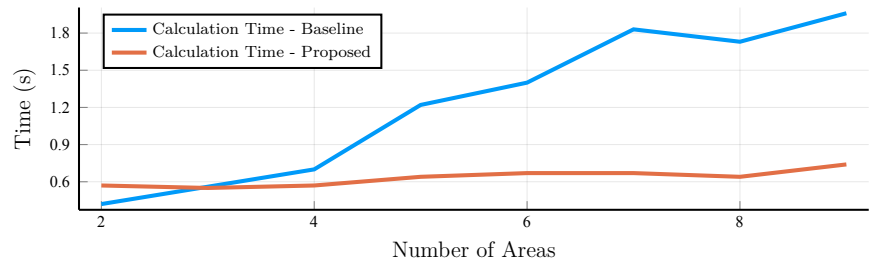

Fig. 6. Comparison of the error in the objective value of the optimal power flow solution (a), and the calculation time (b), for creating the reduced network of the IEEE 118-buses transmission system for different number of areas.

\section{REFERENCES}

[1] S. Wogrin, D. A. Tejada-Arango, S. Pineda, and J. M. Morales, "What time-period aggregation method works best for power system operation models with renewables and storage?" in 2019 International Conference on Smart Energy Systems and Technologies (SEST), Sep. 2019, pp. 1-6.

[2] E. Shayesteh, C. Hamon, M. Amelin, and L. Söder, "Rei method for multi-area modeling of power systems," International Journal of Electrical Power \& Energy Systems, vol. 60, pp. 283 - 292, 2014.

[3] E. Shayesteh, M. Amelin, and L. Soder, "Area equivalents for spinning reserve determination in interconnected power systems," Energy, vol. 88, pp. $907-916,2015$.

[4] E. Shayesteh, B. F. Hobbs, L. Söder, and M. Amelin, "Atc-based system reduction for planning power systems with correlated wind and loads," IEEE Transactions on Power Systems, vol. 30, no. 1, pp. 429-438, Jan 2015.

[5] E. Cotilla-Sanchez, P. D. H. Hines, C. Barrows, S. Blumsack, and M. Patel, "Multi-attribute partitioning of power networks based on electrical distance," IEEE Transactions on Power Systems, vol. 28, no. 4, pp. 4979-4987, Nov 2013.

[6] J. Li, C. Liu, and K. P. Schneider, "Controlled partitioning of a
TABLE I

ACCuracy and Computation Time for the Reduced Power Flow MODEl OF THE IEEE 118 -BUSES TEST SYSTEM FOR DIFFERENT NUMBER OF AREAS.

\begin{tabular}{|l|l|l|l|l|}
\hline \multirow{2}{*}{$\begin{array}{l}\text { ieee 118- } \\
\text { buses }\end{array}$} & $\begin{array}{l}\text { Active Power } \\
\text { Generation }\end{array}$ & $\begin{array}{l}\text { Operational } \\
\text { Cost }\end{array}$ & $\begin{array}{l}\text { Computation } \\
\text { Time } \\
\text { Reduction (\%) }\end{array}$ & $\begin{array}{l}\text { Number } \\
\text { of Areas }\end{array}$ \\
\cline { 2 - 4 } & \multicolumn{2}{|c|}{ Error (\%) } & 71.2 & 2 \\
\hline \hline Scenario 1 & 0.97 & 1.44 & 65.20 & 3 \\
Scenario 2 & 0.53 & 0.92 & 44.77 & 4 \\
Scenario 3 & 0.40 & 0.77 & 34.45 & 6 \\
Scenario 4 & 0.26 & 0.42 & -2.61 & 8 \\
Scenario 5 & 0.38 & 0.24 & \multicolumn{2}{|l}{} \\
\hline
\end{tabular}

TABLE II

ACCURACY AND COMPUTATION TIME FOR THE REDUCED OPTIMAL POWER Flow MOdel of DifFERENT TeSt Systems.

\begin{tabular}{|l|l|l|l|}
\hline $\begin{array}{l}\text { Case } \\
\text { Study }\end{array}$ & $\begin{array}{l}\text { Number of } \\
\text { Areas }\end{array}$ & $\begin{array}{l}\text { Active Power } \\
\text { Generation } \\
\text { Error (\%) }\end{array}$ & $\begin{array}{l}\text { Computation time } \\
\text { Reduction (\%) }\end{array}$ \\
\hline ieee 39-buses & 2 & 0.05 & 64.3 \\
ieee 118-buses & 3 & 0.49 & 66.22 \\
ieee 300-buses & 4 & 1.01 & 58.19 \\
ACTIVSg2000 & 4 & 0.05 & 86.76 \\
\hline
\end{tabular}

power network considering real and reactive power balance," IEEE Transactions on Smart Grid, vol. 1, no. 3, pp. 261-269, Dec 2010.

[7] P. Fortenbacher, T. Demiray, and C. Schaffner, "Transmission network reduction method using nonlinear optimization," in 2018 Power Systems Computation Conference (PSCC), June 2018, pp. 1-7.

[8] D. Klein, C. Spieker, S. Rüberg, V. Liebenau, and C. Rehtanz, "Aggregation of large-scale electrical energy transmission networks," in 2016 IEEE International Energy Conference (ENERGYCON), April 2016, pp. $1-6$.

[9] D. Grosso, R. Gerboni, and D. Cotugno, "Modelling urban transport sector: A methodology based on osemosys model generator," in 2017 IEEE 41st Annual Computer Software and Applications Conference (COMPSAC), vol. 2, July 2017, pp. 754-759.

[10] R. Loulou, U. Remne, A. Kanudia, A. Lehtila, and G. Goldstein. (2005) Documentation for the times model - part i 1-78. [Online]. Available: http://iea-etsap.org/docs/TIMESDoc-Intro.pdf

[11] M. Maruf, "Sector coupling in the north sea region-a review on the energy system modelling perspective," Energies, vol. 12, no. 4298, 2019.

[12] (2019) Project spine. [Online]. Available: http://www.spine-model.org/

[13] M. Marin, E. Rinne, P. Vennström, A. Soininen, and P. Savolainen. (2020) Spine toolbox user guide. [Online]. Available: https://spinetoolbox.readthedocs.io

[14] C. Coffrin, R. Bent, K. Sundar, Y. Ng, and M. Lubin, "Powermodels.jl: An open-source framework for exploring power flow formulations," in 2018 Power Systems Computation Conference (PSCC), June 2018, pp. $1-8$.

[15] (2019) Nrel/powersystems.jl. [Online]. Available: https://github.com/NREL/PowerSystems.j1

[16] L. Thurner, A. Scheidler, F. Schäfer, J. Menke, J. Dollichon, F. Meier, S. Meinecke, and M. Braun, "Pandapower-an open-source python tool for convenient modeling, analysis, and optimization of electric power systems," IEEE Transactions on Power Systems, vol. 33, no. 6, pp. 65106521, Nov 2018.

[17] J. Olauson, M. Marin, and L. Söder, "Creating power system network layouts: A fast parallel algorithm," IEEE Systems Journal, pp. 1-8, 2020.

[18] M. L. Oatts, S. R. Erwin, and J. L. Hart, "Application of the rei equivalent for operations planning analysis of interchange schedules," IEEE Transactions on Power Systems, vol. 5, no. 2, pp. 547-555, May 1990. 